Send your letters to the editor, British Dental

Journal, 64 Wimpole Street, London W1G 8YS E-mail bdj@bda.org

Priority will be given to letters less than 500

words long. Authors must sign the letter,

which may be edited for reasons of space

\section{Format problem}

Sir,- I am becoming increasingly frustrated with the current versions of the BNF (and DPF) and believe that opening a debate on the matter may contribute to the development of improved products. Notwithstanding the excellent pharmacological data contained within the BNF, I find the current hardcopy version infuriating as, on multiple occasions, it refers the reader to another section which then re-refers to yet another section. In my opinion, it is poorly designed in this regard. I would not be surprised if a significant proportion of the high number of prescribing errors among medical professionals were attributable to the current problems navigating the text for drug interaction and other advice (particularly when the user is under pressure). I believe that the e-bnf has a confusing and frustrating navigation system. The web-based system appears to be so light on information that it may represent a medico-legal threat to users. For example, the page which I visited on amoxicillin oral powder at www.bnf.org /bnf/bnf/current/openat/105952.htm, only gives outline information on available preparations. It gave no dosage, indications or ADR information or links on the relevant frame.

In my opinion it should be possible to simply create the whole BNF in a PDF (the editing function could be disabled), RTF or Word format by converting the original file format used to create the hardcopy version. Such file formats would allow users to navigate the document using the simple search, page jump, hypertext and cross-reference functions (as available in the relevant format). Search speeds would not be an issue with the recent generations of computers. I suspect that copyright issues may have prevented the publication of the original document in a simple formatted text file (or more sophisticated format) matching the original. The BNF is supposed to be a key aid to clinicians not a pharmacological data maze. The committee responsible for the document should take a hard look at improving it and the format/s in which it is presented.

\section{P. V. Mc Crory} Radcliffe

\section{R. Macfarlane, Assistant Editor, BNF and} S. M. S. Wagle, Assistant Editor, BNF/DPF respond:

Dr Mc Crory is confusing two different publications. On the one-hand, the British National Formulary (BNF) which includes the Dental List, and on the other, the Dental Practitioners' Formulary (DPF) which includes prescribing information on preparations that dental surgeons can prescribe on Form FP10 (GP14 in Scotland). The Dental List in the BNF is a simple list of preparations which a dental surgeon may prescribe on the NHS; the list is not intended to be used as a resource for prescribing information. There is currently no digital (electronic) version of the DPF. Whereas the UK health departments purchase DPFs for distribution to dental surgeons, there is no arrangement for the publishers to provide a digital version. Despite this, a digital version of the DPF was developed but the uptake was very poor and it was agreed that for the time being no further effort should go into producing a digital DPF.

There are several digital versions of the BNF, all of which include the Dental List. The digital version of the BNF on the Internet (http://BNF.org) is made available free-of-charge, without any restriction on access. The publishers receive no funding for this service and it is provided with the sole purpose of making the information available to healthcare professionals as readily as possible. The BNF website is appreciated by healthcare professionals in the UK and particularly abroad. The editorial team and the software developers are very happy to listen to users' comments and make improvements. In any case, the BNF has embarked on a very ambitious (and resource-intensive) programme of developing its digital offerings. The result of some of this work is already visible in the new-look digital BNF on the website. Further innovative features will follow.
Since the digital versions of the BNF contain the same information as the printed book, we are puzzled by Dr Mc Crory's remark that the 'web based system' is light on information. Dr Mc Crory's example about amoxicillin oral powder relates to the Dental List. The Dental List was never intended to include prescribing information on dental indications, doses, and side-effects. As we say, that information is in the DPF.

More generally, Dr Mc Crory finds the BNF's navigation system confusing. Users can either browse through the hierarchy of chapters (in the traditional way) or locate the information by means of a keyword search. Anyone familiar with Google should feel at home with the latter method. So, for example, a search for 'amoxicillin' produces 34 results, displayed according to order of importance and relevance. The first such result links to the amoxicillin monograph in the BNF, which includes information on indications, cautions, contra-indications, side-effects, and doses. This information is not specific to dentistry because the BNF is intended for a far wider audience.

Dr Mc Crory takes issue with the use of cross-references from one section of the text to another. It is customary for medical reference works of this nature not to repeat information in several places; instead, information is provided in its proper context in one place, with pointers to related information elsewhere. Digital reference works also use this approach.

We recognise that healthcare professionals need to learn how to use BNF resources effectively. We are very willing to listen to constructive comments from our healthcare colleagues and to respond to individual issues regarding the BNF family of digital products. doi: 10.1038/sj.bdj.4810543

\section{Overseas dentists}

Sir,- In a recent interview on GMTV the Chief Dental Officer spelled out the government's plans for the future of NHS dentistry. The government proposes to set up NHS dental clinics staffed by salaried 
dentists. This begs the question as to where they will find these dentists. It is unlikely that they will find the many thousands necessary within the UK or within the existing EU. The ethos of the UK GDP is a self-employed professional. Since very few now want to provide NHS treatment, even in general practice, the likelihood of attracting significant numbers into these positions is remote.

The salaries likely to be offered for these positions may appear attractive to those new EU members who have recognised diplomas but, once here, even they will seek the higher remuneration offered by private practice. What the government needs is a workforce that is tied into these clinics as a condition of working in the UK. It would appear that they have been working on this plan for some time because Duncan Rudkin, deputy registrar of the GDC, said in a speech to the DPB in April 2002, 'Conditional registration was also being considered for non-EU practitioners and for those from within the EU a review is underway on the effects of the current European directives.' The Report of the Registration Review Group published in November 2002 states, 'The DoH is concerned that an over-restrictive interpretation of the Act is preventing overseas qualified dentists from obtaining temporary registration for certain posts in the NHS.' (10.20) That the backbone of NHS dental services should be provided by dentists whose training is deemed inadequate, (some of the new EU countries) or of unknown quality, (non-EU countries), either under temporary registration regulations, or as IQE students without registration, another possibility under the new Dentists Act, is totally unacceptable.

There is still time for the new elected GDC to reverse the decision of the previous, mainly appointed, GDC to allow temporary registrants to work in primary care. If they do not they will be failing both the public and the profession. Incidentally the queue for these jobs is already starting to form. The following is part of a posting on the dentists' web discussion forum GDP-UK, 'Well my friends I am a dentist in India (remember crown jewel etc, your colonial past) and there are thousands of fully qualified dentists (me included) who would love to work anywhere in the UK, government or otherwise (but preferably government). In fact the level of desperation here is such that you would find our dentists ready to work as surgery assistants and hygienists in the UK.' I hope that the GDC will fulfil its duty.

\section{A. Cooper}

London

doi: $10.1038 /$ sj.bdj.4810544

\section{Blood donation and acupuncture}

Sir,-According to the guidelines for blood donation set by the European Council ${ }^{1}$ it is recommended to ask potential donors the following question: Since your last donation or in the previous 12 months have you had [...] acupuncture treatment by anyone other than a registered practitioner?

According to the European Council's recommendation ${ }^{2}$ it is recommended that patients should be excluded from blood donation for one year, if they have received acupuncture by a nonauthorised practitioner.

The rules in England differ slightly from the guidelines set by the European Council. According to the rules applied in England $^{3}$, patients can give blood if the condition for which acupuncture treatment is being received is acceptable, and provided that this has been performed by staff in NHS hospitals or by a medical practitioner, or if the donor presents a valid certificate from an acupuncture practitioner registered with the Council for Acupuncture or the Acupuncture Association of Chartered Physiotherapists. Otherwise they are accepted one year after end of acupuncture treatment.

However, there seems to be some confusion regarding dental practitioners. Thus a number of patients have been refused to give blood after acupuncture by a dental practitioner. Dentists are among other things registered to extract teeth, perform operations in the mouth and give injections. Under those circumstances it is legal for the patient to give blood.

However, if a patient has received acupuncture for a dental related condition by a dental practitioner, the situation is different. In our opinion, dentists should be regarded as registered dental practitioners in the same way as medical practitioners. Therefore clear guidelines would be appreciated.

\section{P. Rosted, Sheffield \\ V. R. K. Jørgensen \\ M. Bundgaard}

Denmark

doi: $10.1038 /$ sj.bdj.4810545

1. Guide to the preparation use and quality assurance of blood components. Recommendation No R (95) 15. 8th ed. Strasbourg: Council of European Publishing 2002.

2. The European Council recommendation 29. June 1998 regarding blood and plasma donors suitability and screening of donor blood in the European Community.

3. Private communication National Blood Service, Trent Centre, Sheffield. 1996 and 2001. 